\title{
Cytokeratin 19-fragments (CYFRA 21-1) as a novel serum biomarker for response and survival in patients with advanced pancreatic cancer
}

\author{
S Boeck ${ }^{*}, 1$, C Wittwer ${ }^{2}$, V Heinemann ${ }^{1}$, M Haas ${ }^{1}$, C Kern ${ }^{1}$, P Stieber ${ }^{2}$, D Nagel ${ }^{2}$ and S Holdenrieder ${ }^{2,3}$ \\ ${ }^{1}$ Department of Internal Medicine III and Comprehensive Cancer Center, Klinikum Grosshadern, Ludwig-Maximilians-University of \\ Munich, Munich, Germany; ${ }^{2}$ Institute of Clinical Chemistry, Klinikum Grosshadern, Ludwig-Maximilians-University of Munich, \\ Munich, Germany and ${ }^{3}$ Institute of Clinical Chemistry and Clinical Pharmacology, University Hospital Bonn, Bonn, Germany
}

Background: CYFRA 21-1 serves as biomarker in several epithelial malignancies. However, its role in pancreatic cancer (PC) has not yet been investigated.

Methods: Within a prospective single-centre study serial blood samples were collected from patients with confirmed advanced PC. Pre-treatment values and weekly measurements of CYFRA 21-1, carbohydrate antigen 19-9 (CA 19-9) and carcinoembryonic antigen (assessed by Elecsys 2010, Roche Diagnostics) during palliative first-line chemotherapy were obtained. Biomarker data were correlated with objective response (determined by RECIST) as well as time to progression (TTP) and overall survival (OS) using uni- and multivariate analyses.

Results: Seventy-eight patients were included, $45 \%$ of these received treatment in prospective clinical trials. Median TTP was 3.9 months, median OS 7.7 months. Pre-treatment CYFRA 21-1 levels were significantly associated with performance status $(P=0.0399)$ and stage of disease $(P=0.0001)$. Marker values before chemotherapy and at the 2-month staging of all three markers were considered significant predictors for objective treatment response. Pre-treatment CYFRA 21-1 levels, as well as CA 19-9 values, could be applied to define subgroups (categorised by tertiles) with a different OS outcome (CYFRA: 14.8 vs 7.1 vs 4.8 months, CA 19-9: 14.2 vs 7.1 vs 5.2 months; P<0.0001). CYFRA 21-1 and CA 19-9 (both as categorised and as continuous variables) showed a highly significant correlation with TTP and OS at nearly all-time points assessed in univariate analysis. In multivariate analysis, only CYFRA 21-1 and performance status were independent predictors for OS.

Conclusions: CYFRA 21-1 may serve as a valuable tool for monitoring treatment response and assessing prognosis in advanced PC.

Pancreatic cancer (PC) is a neoplastic disease known for its unfavourable prognosis and outcome data: the 5-year relative survival rate among patients diagnosed with PC in the United States from 2001 to 2007 was as low as 6\% over all stages (Siegel et al, 2012). In patients with advanced stages of the disease, palliative gemcitabine-based chemotherapy has been the standard of care during the last decade. With the novel FOLFIRINOX regimen, a new treatment option for patients with metastatic PC was recently introduced (Vincent et al 2011; Heinemann et al, 2012). To date, overall survival (OS) remains the standard clinical end point for clinical trials in PC research, and also for the approval of novel drugs (Heinemann et al, 2012). Several efforts have been undertaken to define new (ideally early) surrogate 'biomarker end points' for treatment efficacy and for assessment of 
prognosis. Carbohydrate antigen 19-9 (CA 19-9) is a tumourassociated antigen that equals a sialylated hapten of the Lewis blood group antigen and is present in a broad variety of fetal and adult mucosal cells (Boeck et al, 2006). It is still the most commonly used tumour marker in PC, as many studies in patients with resectable and advanced disease proved CA 19-9 to be a useful tool for evaluation of treatment response as well as prediction of prognosis (Ferrone et al, 2006; Berger et al, 2008; Hess et al, 2008; Reni et al, 2009; Boeck et al, 2010; Humphris et al, 2012). However, a broad variety of other serum and tissue markers presently investigated in PC have not yet been sufficiently validated for routine clinical use (Duffy et al, 2010).

Carcinoembryonic antigen (CEA) is a monomer glycoprotein, which can be found in adult gastrointestinal epithelia, sweat glands, lung epithelia and various epithelial malignancies. In colorectal and lung cancer, pre-therapeutic CEA levels are well established as tumour marker for prediction of time to progression (TTP) and OS; furthermore, CEA kinetics are also known for their important role in the diagnosis of tumour recurrence. CYFRA 21-1 is a fragment of cytokeratin 19, a structure protein and part of intermediate filament proteins necessary for stability of epithelial cells. It is thus expressed in a variety of epithelial cells and has already been shown to be a useful biomarker in lung and breast cancer (Nakata et al, 2004; Holdenrieder et al, 2009; Edelman et al, 2012). Moreover, CYFRA 21-1 was recently shown to be a prognostic relevant marker for OS in metastatic colorectal cancer after selective internal radiation therapy (Fahmueller et al, 2012). At least to our knowledge, clinical data on CYFRA 21-1 in PC are still very rare (Halm et al, 2000; Duffy et al, 2010).

The aim of this prospective, single-centre biomarker study was to investigate the role of CYFRA $21-1$ as serum biomarker in patients with advanced $\mathrm{PC}$ undergoing palliative chemotherapy. To this end, a representative group of $\mathrm{PC}$ patients underwent an extensive biomarker profiling for CYFRA 21-1, CA 19-9 and CEA levels that were assessed centrally before and weekly after the initiation of chemotherapy.

\section{PATIENTS AND METHODS}

Patient population and treatment. Male or female patients with histologically or cytologically confirmed diagnosis of advanced exocrine PC (locally advanced or metastatic stages of disease) were eligible for the current prospective biomarker study. All included patients received palliative chemotherapy. Eighty-three consecutive patients meeting the eligibility criteria were recruited from the 'Pancreas Centre' at the LudwigMaximilians-University of Munich between May 2006 and April 2010. Patients treated outside clinical trials received - based on the decision of the treating medical oncologist SB and $\mathrm{VH}$ - standard gemcitabine or gemcitabine-based chemotherapy until disease progression, unacceptable toxicity or patient refusal (for detailed treatment regimens see Results section). Routine radiological tumour assessment and response evaluation was performed by CT or MRI according to standard RECIST (response evaluation criteria in solid tumors; version 1.0) every 8 weeks. The study was approved by the local ethics committee of the Ludwig-Maximilians-University and all patients gave written informed consent before any study-specific procedure was performed.

Sample collection and assays. Venous blood samples were collected immediately before initiation of first-line chemotherapy (day 0 ) and thereafter weekly on days 7, 14, 21 and 28, and at the time point of the first radiographic staging after 2 months of chemotherapy (day 56). The samples were centrifuged for $15 \mathrm{~min}$ at $3000 \mathrm{~g}$ within $2 \mathrm{~h}$ of venipuncture. Sera were separated manually, aliquoted into microtubes and without any further treatment frozen at $-80{ }^{\circ} \mathrm{C}$ for a maximum of 3 years. For measurements, samples were thawed and assessed in batches containing all samples of one single patient. Measurements of CYFRA 21-1, CA 19-9 and CEA were all performed automatically using the Elecsys 2010 (Roche Diagnostics, Penzberg, Germany) in an electrochemi-luminescence immuno-assay. All assays in this biomarker study were performed blinded to the study end point. In the first step of the assay, the antigens (CYFRA 21-1, CA 19-9 or CEA) are incubated with two types of antigen-specific monoclonal antibodies, one biotinylated, and the other bound to a rutheniumcontaining complex. The resulting sandwich complex of antigen and the two antigen-specific monoclonal antibodies is then bound to streptavidin-coated microparticles via biotin-streptavidin interaction. Next, the mixture is transferred to the measuring cuvette and the antigen-containing complex is magnetically immobilised onto the electrode. After a washing step, chemiluminescence is induced by an electric field and assessed by a photomultiplier. Antigen concentration is determined in the following by use of a calibration curve. CA 19-9all was defined as CA 19-9 values for all patients; CA 19-9syn was defined as CA 19-9 values for patients, who are supposed to be able to synthesise CA 19-9 (i.e., at least one value $>5 \mathrm{U} \mathrm{ml}^{-1}$ ). If not stated otherwise, all analyses were performed with the CA 19-9syn population.

Study design and statistical analysis. This prospective singlecentre biomarker study was designed, conducted and analysed

\begin{tabular}{|c|c|c|}
\hline & Number & Percentage (\%) \\
\hline & 78 & 100 \\
\hline \multicolumn{3}{|c|}{ Gender } \\
\hline $\begin{array}{l}\text { Female } \\
\text { Male }\end{array}$ & $\begin{array}{l}30 \\
48\end{array}$ & $\begin{array}{l}38 \\
62\end{array}$ \\
\hline
\end{tabular}

\section{Stage of disease at study entry}

\begin{tabular}{|l|c|c|}
\hline Synchronous metastases & 51 & 65 \\
Locally advanced disease & 9 & 12 \\
Relapse & 18 & 23
\end{tabular}

\begin{tabular}{|l|c|c|}
\hline \multicolumn{3}{|l|}{ KPS } \\
\hline 100 & 16 & 21 \\
90 & 42 & 54 \\
80 & 13 & 17 \\
70 & 6 & 8 \\
60 & 1 & 1
\end{tabular}

KRAS mutation status (exon 2)

\begin{tabular}{|l|l|l|}
\hline Wild type & 10 & 13 \\
Mutation & 26 & 33 \\
Not assessed & 42 & 54
\end{tabular}

\section{Diabetes mellitus}

\begin{tabular}{|l|l|l|}
\hline Negative & 30 & 38 \\
Known & 13 & 17 \\
Newly diagnosed at study entry & 12 & 15 \\
Not assessed & 23 & 30 \\
\hline
\end{tabular}

Best response by imaging

\begin{tabular}{|l|l|l|}
\hline$C R+P R+S D$ & 42 & 54 \\
$P D$ & 26 & 33 \\
Not assessed & 10 & 13 \\
\hline
\end{tabular}

Abbreviations: $\mathrm{CR}=$ complete remission; $\mathrm{KPS}=$ Karnofsky performance status; $\mathrm{PD}=$ progressive disease; $\mathrm{PR}=$ partial remission; $\mathrm{SD}=$ stable disease. 
according to the 2005 REMARK guidelines ('REporting recommendations for tumour MARKer prognostic studies') as appropriate (McShane et al, 2005). The pre-defined end point of this study was to show a correlation of CYFRA 21-1, CA 19-9 and CEA with efficacy outcome parameters of treatment: objective response determined by RECIST, TTP and OS. Time to progression was defined as the interval between initiation of treatment and occurrence of a documented disease progression; OS was defined as the time interval between initiation of treatment and death from any cause. Concentrations of all measured markers before (day 0), and on days $7,14,21,28$ and 56 (=staging) after start of chemotherapy as well as their differences compared with pretherapeutic levels were considered for statistical evaluation. Significance of differences was tested using the Wilcoxon test for paired samples. Concerning their response to therapy at staging on day 56, patients with complete remission (CR), partial remission (PR) or stable disease (SD) were combined into a 'non-progressive/ disease control' group and compared with patients who suffered from progressive disease. For assessment of significance between marker levels in therapy response groups, the Wilcoxon-MannWhitney test was used. Correlations between marker levels were assessed by the Spearman rank correlation coefficient. Overall survival and TTP were estimated by the Kaplan-Meier method and survival curves were compared using the log-rank test. For this analysis, marker values were separated into tertiles to achieve an equal distribution of the patient number. Marker values were also analysed in univariate Cox regression models, on the one hand as tertiles for the calculation of hazard ratios, and on the other hand as logarithms. In addition, these analyses were done with inclusion of the Karnofsky performance status (KPS) in the models, which was the strongest clinical predictor. All clinical and pre-therapeutical biochemical parameters with a $P$-value $<0.1$ in univariate analysis were included into multivariate Cox regression analysis. To compare the prognostic strength of different models we used the concordance index ( $\mathrm{C}$ index) proposed by Harrell et al (1984). The C index values range from 0.5 to 1 , representing the proportion of concordance in all possible pairs of patients, whereby concordance means that the patient with a more favourable value (closer to 1) has the longer survival time. In addition, exploratory subgroup analyses were performed for patients suffering from diabetes mellitus and also for patients with a known KRAS mutation status. A $P$-value of $<0.05$ was considered statistically significant. All calculations were performed with SAS software (version 9.2, SAS Institute Inc., Cary, NC, USA).

\section{RESULTS}

Patient characteristics. Overall, 83 consecutive PC patients from one German Cancer Centre were included in this biomarker study. Patients started treatment between May 2006 and April 2010; 35 patients $(45 \%)$ in this biomarker study received chemotherapy within a prospective clinical trial. Applied treatment regimens included: gemcitabine plus erlotinib $(n=45)$, single-agent gemcitabine $(n=12)$, gemcitabine plus everolimus $(n=11)$, capecitabine plus erlotinib $(n=9)$, gemcitabine plus axitinib $(n=2)$, singleagent capecitabine $(n=2)$, gemcitabine plus WX-671 $(n=1)$ or nab-paclitaxel $(n=1)$. Owing to missing baseline marker levels, five cases had to be excluded from statistical analysis. Of the 78 remaining evaluable patients, 68 were assessable for objective response by imaging, which was first performed after a median of
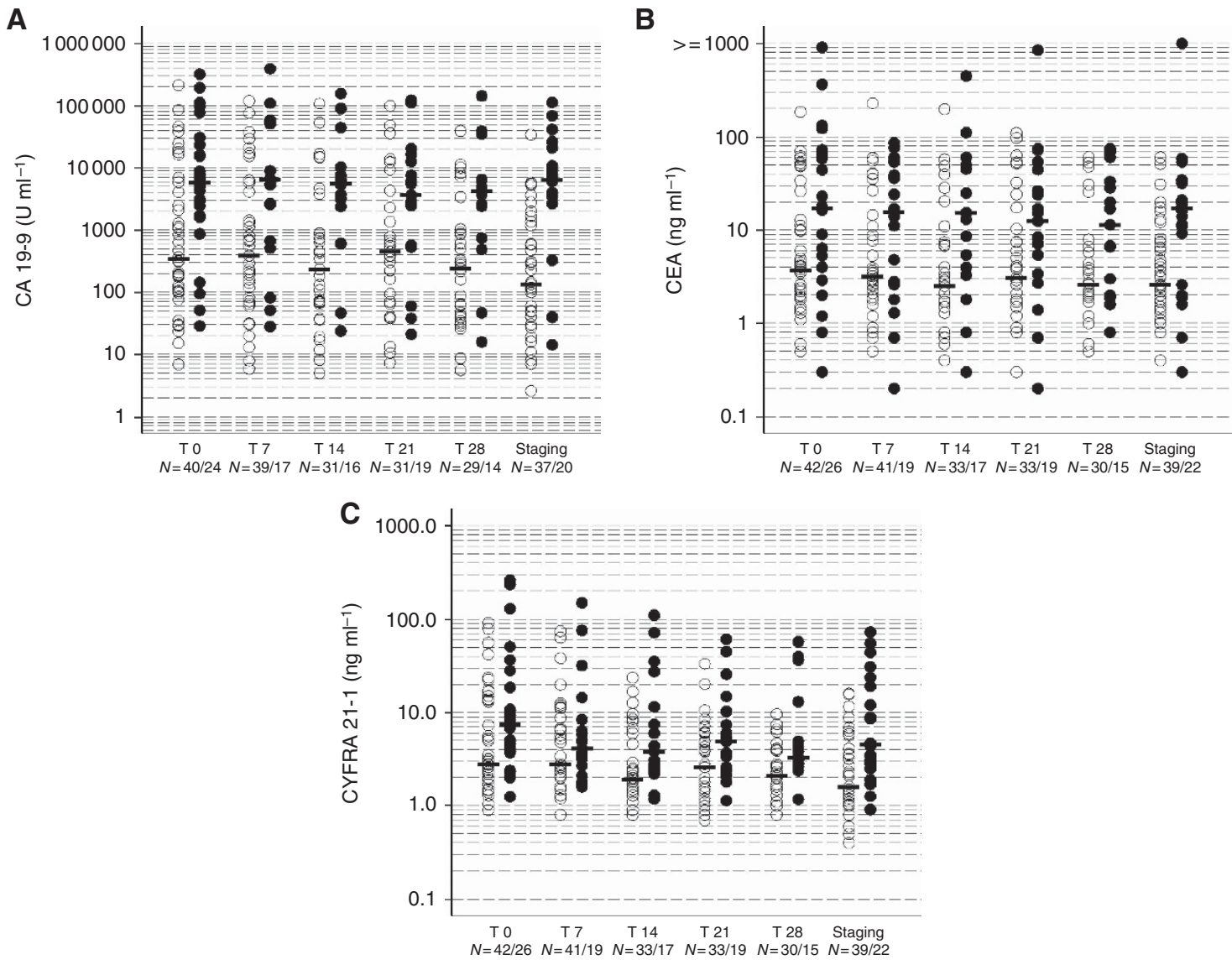

Figure 1. Levels at the time points (T) day $0,7,14,21,28$ and at staging (day 56) in non-progressive and progressive patients (dots with medians) for CA 19-9 (A), CEA (B) and CYFRA 21-1 (C) ( $n=68)$ (Full dots indicating progressive, empty dots non-progressive patients). 
Table 2. Correlation of absolute median biomarker levels (Tx) and marker kinetics (Tx - T0\%) with objective response to chemotherapy ( $n=68)$

\begin{tabular}{|c|c|c|c|c|c|c|c|c|c|}
\hline & & \multicolumn{3}{|c|}{ PD } & \multicolumn{3}{|c|}{$\begin{array}{c}\text { Non-progressive disease } \\
\text { (CR + PR + SD) }\end{array}$} & \multicolumn{2}{|c|}{$\boldsymbol{P}$-value } \\
\hline Marker & Time (days) & $n$ & $T_{x}$ & $\mathrm{~T}_{\mathrm{x}}-\mathrm{T}_{0} \%$ & $n$ & $T_{x}$ & $T_{x}-T_{0} \%$ & $T_{x}$ & $\mathrm{~T}_{\mathrm{x}}-\mathrm{T}_{0} \%$ \\
\hline \multicolumn{10}{|c|}{ CYFRA 21-1 (ng ml${ }^{-1}$ ) } \\
\hline & 0 & 26 & 7.5 & - & 42 & 2.8 & - & 0.011 & - \\
\hline & 7 & 19 & 4.1 & -31.9 & 41 & 2.8 & -8.8 & 0.262 & 0.040 \\
\hline & 14 & 17 & 3.8 & -32.4 & 33 & 1.9 & -31.6 & 0.017 & 0.728 \\
\hline & 21 & 20 & 5.1 & -31.6 & 33 & 2.6 & -40.0 & 0.021 & 0.267 \\
\hline & 28 & 15 & 3.3 & -43.1 & 30 & 2.1 & -28.9 & 0.005 & 0.727 \\
\hline & $\mathrm{S}$ & 23 & 4.6 & -20.8 & 39 & 1.6 & -39.1 & $<0.001$ & 0.311 \\
\hline \multicolumn{10}{|c|}{ CEA $\left(n g \mathrm{ml}^{-1}\right)$} \\
\hline & 0 & 26 & 17.1 & - & 42 & 3.7 & - & 0.008 & - \\
\hline & 7 & 19 & 15.6 & -12.5 & 41 & 3.2 & -10.2 & 0.078 & 0.313 \\
\hline & 14 & 17 & 15.4 & 0.0 & 33 & 2.5 & -22.1 & 0.007 & 0.037 \\
\hline & 21 & 20 & 10.4 & -6.8 & 33 & 3.1 & -27.3 & 0.130 & 0.394 \\
\hline & 28 & 15 & 11.4 & 3.3 & 30 & 2.6 & -26.9 & 0.043 & 0.092 \\
\hline & $\mathrm{S}$ & 23 & 18.1 & 0.0 & 39 & 2.6 & -26.3 & 0.002 & 0.078 \\
\hline \multicolumn{10}{|c|}{ CA $19-9\left(\mathrm{U} \mathrm{ml}^{-1}\right)$} \\
\hline & 0 & 24 & 5810.5 & - & 40 & 341.5 & - & 0.006 & - \\
\hline & 7 & 17 & 6487.0 & -2.4 & 39 & 388.0 & -10.4 & 0.023 & 0.557 \\
\hline & 14 & 16 & 5590.5 & -13.8 & 31 & 232.0 & -32.9 & 0.005 & 0.119 \\
\hline & 21 & 20 & 3657.0 & -20.2 & 31 & 446.0 & -31.2 & 0.020 & 0.259 \\
\hline & 28 & 14 & 4177.0 & -11.5 & 29 & 241.0 & -42.0 & 0.013 & 0.209 \\
\hline & $S$ & 21 & 6428.0 & -17.4 & 37 & 135.0 & -65.2 & $<0.001$ & $<0.001$ \\
\hline
\end{tabular}

57 days after initiation of palliative chemotherapy. At the time of final analysis, 74 of the 78 study patients had experienced disease progression and 73 of 78 had died.

Clinical baseline characteristics are summarised in Table 1. Median age was 65 years (range 41-79) and most patients (65\%) were diagnosed with synchronous metastatic disease at study entry. KRAS mutation status (exon 2, codon 12 and 13) was known in 36 patients (mainly in patients treated in clinical trials) and 25 patients presented with diabetes mellitus at study registration. Twenty-six patients experienced early disease progression based on the 2-month staging interval, whereas 42 patients achieved primary disease control during chemotherapy (1 CR, 4 PR and 37 SD; Table 1). Median TTP for all 78 patients was estimated at 3.9 months (95\% CI 2.3-5.3) and median OS at 7.7 months (95\% CI 6.3-10.0).

Pre-therapeutic biomarker levels. Median baseline CA19-9 levels differed significantly between metastatic and locally advanced disease (3485 vs $\left.129 \mathrm{U} \mathrm{ml}^{-1}, P=0.0126\right)$ and between primary metastatic and recurrent PC (3485 vs $\left.137 \mathrm{U} \mathrm{ml}^{-1}, P=0.005\right)$. Pretherapeutic values of CYFRA 21-1 could also be significantly distinguished between the three groups of primary metastatic, locally advanced and recurrent disease (7.5 vs 1.5 vs $2.6 \mathrm{ng} \mathrm{ml}^{-1}$, $P=0.0001)$. At time of study entry, CYFRA 21-1 was the only marker correlating with good or impaired KPS (KPS 90-100\%: $3.6 \mathrm{ng} \mathrm{ml}^{-1}$ vs $\mathrm{KPS}<90 \%$ : $9.8 \mathrm{ng} \mathrm{ml}^{-1}, P=0.0399$ ). CYFRA 21-1 levels did not differ significantly between patients with newly diagnosed diabetes mellitus and patients without impaired glucose tolerance in subgroup analyses (median 3.9 vs $7.4 \mathrm{ng} \mathrm{ml}^{-1}$, $P=0.437)$. None of the three assessed biomarkers correlated with the KRAS exon 2 mutation status. CYFRA 21-1, CA 19-9 and CEA showed highly significant correlations $(P<0.0001)$ with each other on day 0 before onset of systemic chemotherapy (Supplementary Table S1).

Courses of biomarkers during chemotherapy. The course of the median biomarker levels for CYFRA 21-1, CA 19-9 and CEA (grouped with regard to 'progressive' vs 'disease control' patients) is illustrated in Figure 1 and Table 2. All three markers showed a significant correlation not only before onset but also at all-time points in the course of chemotherapy (Supplementary Table S1). Both CA 19-9 subgroups (CA 19-9all and CA 19-9syn) correlated significantly with CEA ( $P$-values ranging from $<0.0001$ to 0.016 for CA19-9all at day 28) and CYFRA21-1 ( $P$-value ranging from $<0.0001$ to 0.003 for CA19-9all at day 28) at all assessed time points.

Correlation of biomarkers with response. Data from 68 evaluable patients on the correlation of absolute median biomarker levels and marker kinetics with objective response to chemotherapy are summarised in Table 2.

Baseline levels (prediction of response). Pre-therapeutic median values of all three markers were significant predictors of treatment response. Baseline CA 19-9 levels in patients suffering from 
Table 3a. Correlation of serial biomarker levels with TTP

Time to progression

\begin{tabular}{|l|c|c|c|c|c|c|c|c}
\hline Time (days) & $\begin{array}{c}\text { Median TTP } \\
\text { (months) }(\mathrm{Cl})\end{array}$ & $\begin{array}{c}\boldsymbol{P} \text { (log } \\
\text { rank) }\end{array}$ & $\begin{array}{c}\text { Hazard } \\
\text { ratio }(\mathrm{Cl})\end{array}$ & $\boldsymbol{P}(\mathrm{Cox})$ & C index (Cl) & $\begin{array}{c}\text { Hazard ratio } \\
\text { (Cl) (adjusted } \\
\text { for KPS) }\end{array}$ & $\boldsymbol{P}(\mathrm{Cox})$ & $\mathrm{C}$ index (Cl) \\
\hline
\end{tabular}

do

\begin{tabular}{|c|c|c|c|c|c|c|c|c|}
\hline CYFRA 21-1 & & $<0.001$ & & & $0.652(0.592-0.712)$ & & & $0.713(0.658-0.767)$ \\
\hline $\begin{array}{l}<2.7 \\
2.7-10 \\
>10\end{array}$ & $\begin{array}{l}9.8(6.0-19.0) \\
2.2(1.6-3.9) \\
2.7(1.6-4.6)\end{array}$ & & $\begin{array}{l}3.0(1.7-5.5) \\
3.3(1.8-6.0)\end{array}$ & $\begin{array}{l}<0.001 \\
<0.001\end{array}$ & & $\begin{array}{l}3.4(1.9-6.2) \\
3.6(1.9-6.7)\end{array}$ & $\begin{array}{l}<0.001 \\
<0.001\end{array}$ & \\
\hline CEA & & $<0.001$ & & & $0.633(0.571-0.694)$ & & & $0.695(0.6350 .755)$ \\
\hline $\begin{array}{l}<2.9 \\
2.9-15 \\
>15\end{array}$ & $\begin{array}{l}6.5(3.9-17.9) \\
3.8(2.0-6.2) \\
2.1(1.8-3.6)\end{array}$ & & $\begin{array}{l}1.9(1.1-3.4) \\
3.0(1.6-5.5)\end{array}$ & $\begin{array}{l}0.026 \\
<0.001\end{array}$ & & $\begin{array}{l}2.4(1.3-4.3) \\
3.3(1.8-6.2)\end{array}$ & $\begin{array}{c}0.005 \\
<0.001\end{array}$ & \\
\hline CA 19-9 & & 0.016 & & & $0.625(0.555-0.696)$ & & & $0.675(0.614-0.736)$ \\
\hline $\begin{aligned} & <200 \\
& 200-6600 \\
> & 6600\end{aligned}$ & $\begin{array}{l}6.0(3.9-17.9) \\
3.7(2.0-6.9) \\
2.1(1.5-3.9)\end{array}$ & & $\begin{array}{l}1.8(1.0-3.3) \\
2.3(1.3-4.3)\end{array}$ & $\begin{array}{c}0.05 \\
0.006\end{array}$ & & $\begin{array}{l}2.0(1.1-3.7) \\
2.6(1.4-4.8)\end{array}$ & $\begin{array}{l}0.022 \\
0.003\end{array}$ & \\
\hline
\end{tabular}

d7

\begin{tabular}{|c|c|c|c|c|c|c|c|c|}
\hline CYFRA 21-1 & & 0.0046 & & & $0.623(0.553-0.694)$ & & & $0.651(0.574-0.727)$ \\
\hline$<2.5$ & $7.1(4.1-17.9)$ & & & & & & & \\
\hline $2.5-6$ & $3.9(2.0-6.9)$ & & $1.8(1.0-3.3)$ & 0.061 & & $1.8(0.9-3.3)$ & 0.075 & \\
\hline$>6$ & $3.0(1.6-5.0)$ & & $2.8(1.5-5.3)$ & 0.002 & & $2.8(1.5-5.4)$ & 0.002 & \\
\hline CEA & & 0.0183 & & & $0.610(0.535-0.684)$ & & & $0.657(0.590-0.724)$ \\
\hline$<2.6$ & $6.9(3.9-17.9)$ & & & & & & & \\
\hline $2.6-14$ & $4.1(3.0-6.2)$ & & $1.3(0.7-2.5)$ & 0.344 & & $1.7(0.9-3.1)$ & 0.122 & \\
\hline$>14$ & $2.1(1.8-5.0)$ & & $2.4(1.3-4.4)$ & 0.007 & & $2.7(1.4-5.1)$ & 0.002 & \\
\hline CA 19-9 & & 0.0021 & & & $0.639(0.564-0.713)$ & & & $0.670(0.609-0.731)$ \\
\hline$<200$ & $6.2(3.9-19.0)$ & & & & & & & \\
\hline $200-6400$ & $5.2(2.7-7.6)$ & & $1.6(0.8-3.0)$ & 0.167 & & $1.8(0.9-3.5)$ & 0.072 & \\
\hline$>6400$ & $2.1(1.6-3.9)$ & & $3.1(1.6-6.1)$ & 0.001 & & $3.4(1.7-6.7)$ & 0.001 & \\
\hline
\end{tabular}

\begin{tabular}{|c|c|c|c|c|c|c|c|c|}
\hline \multicolumn{9}{|l|}{ d14 } \\
\hline CYFRA 21-1 & & 0.0081 & & & $0.646(0.575-0.718)$ & & & $0.655(0.579-0.732)$ \\
\hline$<1.9$ & $10.3(4.6-21.4)$ & & & & & & & \\
\hline $1.9-6$ & $3.9(1.6-6.2)$ & & $1.9(1.0-3.7)$ & 0.055 & & $2.3(1.2-4.7)$ & 0.018 & \\
\hline$>6$ & $2.8(1.2-5.3)$ & & $2.9(1.4-5.9)$ & 0.003 & & $3.2(1.6-6.7)$ & 0.001 & \\
\hline CEA & & 0.0017 & & & $0.626(0.549-0.703)$ & & & $0.658(0.583-0.733)$ \\
\hline$<2.1$ & $8.0(3.4-17.9)$ & & & & & & & \\
\hline $2.1-11$ & $6.1(3.7-9.7)$ & & $0.9(0.5-1.8)$ & 0.80 & & $1.1(0.5-2.2)$ & 0.849 & \\
\hline$>11$ & $1.8(1.3-3.6)$ & & $2.7(1.4-5.4)$ & 0.003 & & $3.0(1.5-6.1)$ & 0.002 & \\
\hline CA 19-9 & & 0.0232 & & & $0.636(0.556-0.717)$ & & & $0.650(0.572-0.728)$ \\
\hline$<150$ & $6.1(3.7-19.0)$ & & & & & & & \\
\hline $150-4000$ & $5.6(1.9-9.7)$ & & $1.4(0.7-2.8)$ & 0.346 & & $1.4(0.7-2.7)$ & 0.387 & \\
\hline$>4000$ & $1.9(1.2-3.9)$ & & $2.6(1.3-5.2)$ & 0.009 & & $2.5(1.2-5.2)$ & 0.011 & \\
\hline \multicolumn{9}{|l|}{ d21 } \\
\hline CYFRA 21-1 & & $<0.001$ & & & $0.674(0.605-0.743)$ & & & $0.716(0.652-0.780)$ \\
\hline$<2.1$ & $9.7(4.6-19.0)$ & & & & & & & \\
\hline $2.1-6$ & $3.4(2.0-4.6)$ & & $2.7(1.3-5.4)$ & 0.005 & & $2.7(1.3-5.5)$ & 0.005 & \\
\hline$>6$ & $2.1(1.4-4.6)$ & & $4.7(2.2-9.9)$ & $<0.001$ & & $3.9(1.8-8.3)$ & 0.001 & \\
\hline CEA & & 0.1314 & & & $0.578(0.501-0.654)$ & & & $0.685(0.623-0.748)$ \\
\hline$<2.5$ & $5.3(2.5-11.9)$ & & & & & & & \\
\hline $2.5-9$ & $4.0(1.5-6.9)$ & & $1.1(0.6-2.2)$ & 0.742 & & $1.1(0.6-2.1)$ & 0.778 & \\
\hline$>9$ & $2.2(1.8-5.0)$ & & $1.9(1.0-3.6)$ & 0.064 & & $1.7(0.9-3.3)$ & 0.132 & \\
\hline
\end{tabular}




\section{Table 3a. (Continued)}

Time to progression

\begin{tabular}{|c|c|c|c|c|c|c|c|c|}
\hline Time (days) & $\begin{array}{l}\text { Median TTP } \\
\text { (months) (Cl) }\end{array}$ & $\begin{array}{l}P \text { (log } \\
\text { rank) }\end{array}$ & $\begin{array}{l}\text { Hazard } \\
\text { ratio }(\mathrm{Cl})\end{array}$ & $P($ Cox $)$ & $\mathrm{C}$ index $(\mathrm{Cl})$ & $\begin{array}{l}\text { Hazard ratio } \\
\text { (CI) (adjusted } \\
\text { for KPS) }\end{array}$ & $P(\mathrm{Cox})$ & $\mathrm{C}$ index $(\mathrm{Cl})$ \\
\hline CA 19-9 & & 0.0492 & & & $0.615(0.529-0.70)$ & & & $0.687(0.626-0.748)$ \\
\hline$<310$ & $5.9(3.4-11.9)$ & & & & & & & \\
\hline $310-4000$ & $3.9(1.8-5.3)$ & & $1.9(0.9-3.7)$ & 0.071 & & $1.6(0.8-3.2)$ & 0.200 & \\
\hline$>4000$ & $2.1(1.6-4.6)$ & & $2.3(1.1-4.5)$ & 0.022 & & $1.8(0.9-3.7)$ & 0.120 & \\
\hline
\end{tabular}

\section{d28}

\begin{tabular}{|c|c|c|c|c|c|c|c|c|}
\hline CYFRA 21-1 & & 0.0674 & & & $0.623(0.544-0.703)$ & & & $0.637(0.554-0.719)$ \\
\hline$<2.1$ & $6.2(3.7-19.0)$ & & & & & & & \\
\hline $2.1-3.8$ & $3.9(1.6-5.8)$ & & $1.6(0.8-3.3)$ & 0.169 & & $1.9(0.9-4.0)$ & 0.077 & \\
\hline$>3.8$ & $2.3(1.6-5.3)$ & & $2.3(1.1-4.6)$ & 0.025 & & $2.3(1.1-4.7)$ & 0.024 & \\
\hline CEA & & 0.1012 & & & $0.574(0.485-0.662)$ & & & $0.639(0.552-0.726)$ \\
\hline$<2.0$ & $5.3(1.6-9.7)$ & & & & & & & \\
\hline $2.0-6$ & $5.0(2.3-11.5)$ & & $0.7(0.3-1.5)$ & 0.378 & & $0.6(0.3-1.4)$ & 0.235 & \\
\hline$>6$ & $2.3(1.8-5.1)$ & & $1.6(0.8-3.2)$ & 0.216 & & $1.5(0.7-3.1)$ & 0.265 & \\
\hline CA 19-9 & & 0.0961 & & & $0.621(0.532-0.710)$ & & & $0.661(0.582-0.741)$ \\
\hline$<100$ & $5.9(3.4-19.0)$ & & & & & & & \\
\hline $100-2500$ & $5.0(2.3-7.1)$ & & $1.6(0.8-3.3)$ & 0.217 & & $1.7(0.8-3.6)$ & 0.150 & \\
\hline$>2500$ & $2.1(1.6-3.9)$ & & $2.2(1.1-4.6)$ & 0.035 & & $2.4(1.1-5.1)$ & 0.024 & \\
\hline
\end{tabular}

\section{d56}

\begin{tabular}{|c|c|c|c|c|c|c|c|c|}
\hline CYFRA 21-1 & & $<0.001$ & & & $0.672(0.613-0.731)$ & & & $0.697(0.636-0.759)$ \\
\hline$<1.7$ & $7.1(6.0-12.0)$ & & & & & & & \\
\hline $1.7-5$ & $2.6(1.8-5.8)$ & & $2.0(1.1-3.7)$ & 0.024 & & $2.4(1.3-4.4)$ & 0.007 & \\
\hline$>5$ & $2.1(1.4-3.6)$ & & $3.2(1.7-6.1)$ & $<0.001$ & & $3.0(1.6-5.7)$ & 0.001 & \\
\hline CEA & & $<0.001$ & & & $0.641(0.572-0.710)$ & & & $0.725(0.664-0.786)$ \\
\hline$<2.5$ & $7.0(4.6-17.9)$ & & & & & & & \\
\hline $2.5-13$ & $5.5(2.7-7.6)$ & & $1.5(0.8-2.8)$ & 0.173 & & $1.3(0.7-2.4)$ & 0.394 & \\
\hline$>13$ & $2.0(1.6-3.6)$ & & $3.3(1.7-6.3)$ & $<0.001$ & & $2.9(1.5-5.7)$ & 0.002 & \\
\hline CA 19-9 & & $<0.001$ & & & $0.688(0.625-0.751)$ & & & $0.719(0.663-0.776)$ \\
\hline$<120$ & $8.4(3.7-19.0)$ & & & & & & & \\
\hline $120-3960$ & $5.8(2.7-7.7)$ & & $1.5(0.8-2.9)$ & 0.205 & & $1.6(0.8-3.1)$ & 0.144 & \\
\hline$>3960$ & $1.9(1.4-2.1)$ & & $4.6(2.3-9.3)$ & $<0.001$ & & $4.4(2.1-8.9)$ & $<0.001$ & \\
\hline
\end{tabular}

Abbreviations: CA 19-9 = Carbohydrate antigen 19-9; CEA = carcinoembryonic antigen; $\mathrm{Cl}=95 \%$ confidence interval; $d=$ day; $K P S=$ Karnofsky performance status; TTP $=$ time to progression The bold entries indicate the highest $C$ index (assessed at each specific time point for all 3 markers.

progressive disease (median $5811 \mathrm{U} \mathrm{ml}^{-1}$, range 29-320000) were elevated compared with patients with non-progressive disease (median $342 \mathrm{U} \mathrm{ml}^{-1}$, range $7-214000, P=0.006$ ). Median CEA baseline levels were higher in progressive $\left(17.1 \mathrm{ng} \mathrm{ml}^{-1}\right.$, range $0.3-908.0$ ) than in non-progressive cases $\left(3.7 \mathrm{ng} \mathrm{ml}^{-1}\right.$, range $0.5-185, P=0.008$ ) and CYFRA 21-1 levels were also significantly higher in progressive patients (median $7.5 \mathrm{ng} \mathrm{ml}^{-1}$, range 1.3-263) than in patients with disease control (median $2.8 \mathrm{ng} \mathrm{ml}^{-1}$, range $0.9-91.5, P=0.01)$.

Marker levels during the first 2 months of chemotherapy (early estimation of response). Absolute levels of CYFRA 21-1, CA 19-9 and CEA showed an excellent correlation with treatment response at almost all assessed time points (see Table 2). On day 14, CEA kinetics could best distinguish between the two response groups ( $0 \%$ decrease in the progressive $v s 22 \%$ decrease in the nonprogressive group, $P=0.037$ ). At the time of staging (day 56), absolute levels of each analysed marker again showed a significant correlation with objective response. Median CA 19-9 levels in patients suffering from progressive disease $\left(6428 \mathrm{U} \mathrm{ml}^{-1}\right.$, range 14-113000) were exceedingly higher than those in patients with disease control (135 $\mathrm{U} \mathrm{ml}^{-1}$, range 2.6-34047, $P<0.0001$ ). A similar discriminatory power was detected for CYFRA 21-1 on day 56 (4.6 vs $\left.1.6 \mathrm{ng} \mathrm{ml}^{-1}, P<0.001\right)$.

Correlation of biomarkers with prognosis. The impact of clinical parameters on efficacy end points is shown in Supplementary Table S2. As expected, KPS was significantly associated with both TTP and OS $(P<0.001)$, and stage of disease was correlated with OS $(P=0.03)$. KPS had the highest $C$ index in this model, with 0.611 (95\% CI $0.555-0.668)$ for TTP and 0.619 (95\% 0.562-0.676) for OS, indicating KPS as the clinical variable with the strongest impact on outcome.

Correlation of baseline biomarker values and of levels during therapy with outcome in univariate analysis. For this complex analysis, all biomarker levels on day $0,7,14,21,28$ and 56 were first categorised into tertiles to ensure equal distribution of patient numbers (see Tables $3 \mathrm{a}$ and $3 \mathrm{~b}$ ). Concerning baseline levels, all three markers had a significant impact on TTP and OS. Tables 3a and $3 \mathrm{~b}$ show data analysed by the log-rank test, by an (unadjusted) univariate Cox model and by a Cox model that was adjusted for 
Table 3b. Correlation of serial biomarker levels with OS

\section{Overall survival}

\begin{tabular}{|c|c|c|c|c|c|c|c|c|}
\hline Time (days) & $\begin{array}{l}\text { Median OS } \\
\text { (months) (CI) }\end{array}$ & $\begin{array}{l}P \text { (log } \\
\text { rank) }\end{array}$ & $\begin{array}{l}\text { Hazard } \\
\text { ratio }(\mathrm{Cl})\end{array}$ & $P($ Cox $)$ & $\mathrm{C}$ index $(\mathrm{Cl})$ & $\begin{array}{l}\text { Hazard ratio } \\
\text { (CI) (adjusted } \\
\text { for KPS) }\end{array}$ & $P($ Cox $)$ & $\mathrm{C}$ index $(\mathrm{Cl})$ \\
\hline \multicolumn{9}{|l|}{ do } \\
\hline CYFRA 21-1 & & $<0.001$ & & & $0.693(0.637-0.748)$ & & & $0.746(0.694-0.799)$ \\
\hline $\begin{array}{l}<2.7 \\
2.7-10 \\
>10\end{array}$ & $\begin{array}{c}14.8(8.4-23.7) \\
7.1(5.4-9.5) \\
4.8(3.2-6.3)\end{array}$ & & $\begin{array}{l}2.9(1.6-5.4) \\
5.0(2.6-9.5)\end{array}$ & $\begin{array}{l}0.001 \\
<0.001\end{array}$ & & $\begin{array}{c}3.1(1.6-5.8) \\
5.5(2.9-10.8)\end{array}$ & $\begin{array}{l}0.001 \\
<0.001\end{array}$ & \\
\hline CEA & & $<0.001$ & & & $0.618(0.553-0.684)$ & & & $0.693(0.624-0.761)$ \\
\hline $\begin{array}{l}<2.9 \\
2.9-15 \\
>15\end{array}$ & $\begin{array}{c}12.9(8.2-23.7) \\
7.1(4.4-8.4) \\
6.3(4.6-8.2)\end{array}$ & & $\begin{array}{l}2.6(1.4-4.7) \\
3.0(1.6-5.7)\end{array}$ & $\begin{array}{l}0.002 \\
0.001\end{array}$ & & $\begin{array}{l}3.1(1.7-5.8) \\
3.1(1.6-5.9)\end{array}$ & $\begin{array}{l}<0.001 \\
0.001\end{array}$ & \\
\hline CA 19-9 & & $<0.001$ & & & $0.660(0.589-0.731)$ & & & $0.709(0.647-0.772)$ \\
\hline $\begin{array}{l}<200 \\
200-6600 \\
>6600\end{array}$ & $\begin{array}{c}14.2(9.4-24.3) \\
7.1(5.8-8.3) \\
5.2(2.9-7.7)\end{array}$ & & $\begin{array}{l}3.0(1.6-5.7) \\
3.6(1.9-6.9)\end{array}$ & $\begin{array}{c}0.001 \\
<0.001\end{array}$ & & $\begin{array}{l}3.1(1.6-6.0) \\
4.3(2.2-8.5)\end{array}$ & $\begin{array}{l}0.001 \\
<0.001\end{array}$ & \\
\hline \multicolumn{9}{|l|}{ d7 } \\
\hline CYFRA 21-1 & & $<0.001$ & & & $0.683(0.623-0.744)$ & & & $0.713(0.647-0.780)$ \\
\hline $\begin{array}{l}<2.5 \\
2.5-6 \\
>6\end{array}$ & $\begin{array}{c}14.2(8.4-23.3) \\
9.4(6.6-10.3) \\
5.0(3.2-7.6)\end{array}$ & & $\begin{array}{l}2.2(1.1-4.1) \\
4.6(2.3-9.0)\end{array}$ & $\begin{array}{l}0.021 \\
<0.001\end{array}$ & & $\begin{array}{l}2.1(1.1-4.1) \\
4.8(2.4-9.6)\end{array}$ & $\begin{array}{c}0.028 \\
<0.001\end{array}$ & \\
\hline CEA & & 0.021 & & & $0.607(0.535-0.680)$ & & & $0.679(0.607-0.750)$ \\
\hline $\begin{array}{l}<2.6 \\
2.6-14 \\
>14\end{array}$ & $\begin{array}{c}11.9(8.2-19.0) \\
8.3(7.1-10.8) \\
6.4(4.7-8.2)\end{array}$ & & $\begin{array}{l}1.4(0.8-2.5) \\
2.4(1.3-4.6)\end{array}$ & $\begin{array}{l}0.296 \\
0.007\end{array}$ & & $\begin{array}{l}1.8(0.9-3.3) \\
3.0(1.5-5.9)\end{array}$ & $\begin{array}{l}0.084 \\
0.001\end{array}$ & \\
\hline CA 19-9 & & $<0.001$ & & & $0.658(0.585-0.731)$ & & & $0.705(0.641-0.769)$ \\
\hline $\begin{array}{l}<200 \\
200-6400 \\
>6400\end{array}$ & $\begin{array}{c}14.8(9.4-24.3) \\
7.7(6.4-11.9) \\
6.0(3.0-9.5)\end{array}$ & & $\begin{array}{l}2.1(1.1-4.0) \\
3.9(1.9-8.0)\end{array}$ & $\begin{array}{l}0.028 \\
<0.001\end{array}$ & & $\begin{array}{l}2.6(1.3-5.1) \\
4.2(2.1-8.7)\end{array}$ & $\begin{array}{l}0.006 \\
<0.001\end{array}$ & \\
\hline \multicolumn{9}{|l|}{ d14 } \\
\hline CYFRA 21-1 & & $<0.001$ & & & $0.677(0.610-0.745)$ & & & $0.687(0.615-0.759)$ \\
\hline $\begin{array}{l}<1.9 \\
1.9-6 \\
>6\end{array}$ & $\begin{array}{c}16.1(8.4-23.3) \\
10.1(6.2-14.8) \\
5.9(3.5-7.6)\end{array}$ & & $\begin{array}{l}1.6(0.8-3.1) \\
4.4(2.1-9.2)\end{array}$ & $\begin{array}{c}0.197 \\
<0.001\end{array}$ & & $\begin{array}{c}1.9(0.9-3.8) \\
4.8(2.3-10.4)\end{array}$ & $\begin{array}{c}0.084 \\
<0.001\end{array}$ & \\
\hline CEA & & 0.0223 & & & $0.609(0.531-0.686)$ & & & $0.650(0.575-0.725)$ \\
\hline $\begin{array}{l}<2.1 \\
2.1-11 \\
>11\end{array}$ & $\begin{array}{c}12.9(7.0-19.0) \\
9.4(7.1-17.9) \\
6.4(4.7-8.3)\end{array}$ & & $\begin{array}{l}1.1(0.6-2.2) \\
2.4(1.2-4.9)\end{array}$ & $\begin{array}{l}0.787 \\
0.013\end{array}$ & & $\begin{array}{l}1.3(0.6-2.7) \\
2.6(1.3-5.4)\end{array}$ & $\begin{array}{l}0.455 \\
0.008\end{array}$ & \\
\hline CA 19-9 & & 0.0032 & & & $0.648(0.572-0.724)$ & & & $0.671(0.593-0.749)$ \\
\hline $\begin{array}{l}<150 \\
150-4000 \\
>4000\end{array}$ & $\begin{array}{c}15.5(8.2-25.0) \\
10.3(6.8-13.9) \\
6.4(3.6-8.3)\end{array}$ & & $\begin{array}{l}1.9(0.9-3.8) \\
3.5(1.6-7.4)\end{array}$ & $\begin{array}{l}0.090 \\
0.001\end{array}$ & & $\begin{array}{l}1.9(0.9-3.9) \\
3.5(1.6-7.4)\end{array}$ & $\begin{array}{l}0.078 \\
0.002\end{array}$ & \\
\hline \multicolumn{9}{|l|}{$\mathrm{d} 21$} \\
\hline CYFRA 21-1 & & $<0.001$ & & & $0.684(0.621-0.748)$ & & & $0.733(0.671-0.795)$ \\
\hline $\begin{array}{l}<2.1 \\
2.1-6 \\
>6\end{array}$ & $\begin{array}{c}11.9(7.1-19.0) \\
7.1(5.4-10.0) \\
4.6(2.0-5.8)\end{array}$ & & $\begin{array}{l}1.5(0.8-2.9) \\
4.2(2.1-8.6)\end{array}$ & $\begin{array}{c}0.251 \\
<0.001\end{array}$ & & $\begin{array}{l}1.4(0.7-2.8) \\
3.5(1.7-7.3)\end{array}$ & $\begin{array}{l}0.291 \\
0.001\end{array}$ & \\
\hline CEA & & 0.1853 & & & $0.581(0.510-0.653)$ & & & $0.701(0.637-0.766)$ \\
\hline $\begin{array}{l}<2.5 \\
2.5-9 \\
>9\end{array}$ & $\begin{aligned} 10.2 & (5.6-16.1) \\
7.1 & (3.0-10.8) \\
5.9 & (3.6-8.2)\end{aligned}$ & & $\begin{array}{l}1.3(0.7-2.5) \\
1.9(0.9-3.7)\end{array}$ & $\begin{array}{l}0.464 \\
0.073\end{array}$ & & $\begin{array}{l}1.2(0.6-2.4) \\
2.0(1.0-3.9)\end{array}$ & $\begin{array}{l}0.514 \\
0.050\end{array}$ & \\
\hline
\end{tabular}


Table 3b. (Continued)

\begin{tabular}{|c|c|c|c|c|c|c|c|c|}
\hline \multicolumn{9}{|c|}{ Overall survival } \\
\hline Time (days) & $\begin{array}{l}\text { Median OS } \\
\text { (months) (Cl) }\end{array}$ & $\begin{array}{l}P \text { (log } \\
\text { rank) }\end{array}$ & $\begin{array}{l}\text { Hazard } \\
\text { ratio }(\mathrm{Cl})\end{array}$ & $P($ Cox $)$ & $\mathrm{C}$ index $(\mathrm{Cl})$ & $\begin{array}{l}\text { Hazard ratio } \\
\text { (CI) (adjusted } \\
\text { for KPS) }\end{array}$ & $P($ Cox $)$ & $\mathrm{C}$ index $(\mathrm{Cl})$ \\
\hline CA 19-9 & & 0.0104 & & & $0.644(0.569-0.720)$ & & & $0.722(0.656-0.787)$ \\
\hline $\begin{array}{l}<310 \\
310-4000 \\
>4000\end{array}$ & $\begin{array}{c}11.4(7.1-19.0) \\
7.0(4.6-10.2) \\
5.8(3.0-6.6)\end{array}$ & & $\begin{array}{l}2.0(1.0-4.1) \\
2.9(1.4-5.9)\end{array}$ & $\begin{array}{l}0.046 \\
0.004\end{array}$ & & $\begin{array}{l}1.9(1.0-3.9) \\
2.4(1.2-5.0)\end{array}$ & $\begin{array}{l}0.066 \\
0.020\end{array}$ & \\
\hline \multicolumn{9}{|l|}{ d28 } \\
\hline CYFRA 21-1 & & 0.0096 & & & $0.637(0.552-0.722)$ & & & $0.651(0.564-0.739)$ \\
\hline $\begin{array}{l}<2.1 \\
2.1-3.8 \\
>3.8\end{array}$ & $\begin{array}{c}11.9(6.8-19.0) \\
8.3(6.2-11.3) \\
5.8(3.0-7.7)\end{array}$ & & $\begin{array}{l}1.2(0.6-2.4) \\
2.8(1.3-5.8)\end{array}$ & $\begin{array}{l}0.663 \\
0.006\end{array}$ & & $\begin{array}{l}1.3(0.6-2.6) \\
2.9(1.4-6.1)\end{array}$ & $\begin{array}{l}0.499 \\
0.005\end{array}$ & \\
\hline CEA & & 0.2343 & & & $0.545(0.446-0.644)$ & & & $0.621(0.571-0.725)$ \\
\hline $\begin{array}{l}<2.0 \\
2.0-6 \\
>6\end{array}$ & $\begin{array}{l}9.4(5.4-11.9) \\
9.25(4.6-23.3) \\
6.8(5.8-8.4)\end{array}$ & & $\begin{array}{l}0.7(0.3-1.4) \\
1.3(0.6-2.6)\end{array}$ & $\begin{array}{l}0.264 \\
0.537\end{array}$ & & $\begin{array}{l}0.6(0.3-1.2) \\
1.2(0.6-2.4)\end{array}$ & $\begin{array}{l}0.161 \\
0.665\end{array}$ & \\
\hline CA 19-9 & & 0.0487 & & & $0.616(0.526-0.705)$ & & & $0.662(0.585-0.738)$ \\
\hline $\begin{array}{l}<100 \\
100-2500 \\
>2500\end{array}$ & $\begin{array}{c}13.3(5.6-24.3) \\
7.4(4.7-10.8) \\
6.4(4.6-7.7)\end{array}$ & & $\begin{array}{l}1.7(0.8-3.6) \\
2.5(1.2-5.5)\end{array}$ & $\begin{array}{l}0.143 \\
0.017\end{array}$ & & $\begin{array}{l}1.9(0.9-4.0) \\
2.5(1.1-5.4)\end{array}$ & $\begin{array}{l}0.091 \\
0.021\end{array}$ & \\
\hline \multicolumn{9}{|l|}{ d56 } \\
\hline CYFRA 21-1 & & $<0.001$ & & & $0.681(0.620-0.743)$ & & & $0.714(0.648-0.779)$ \\
\hline $\begin{array}{l}<1.7 \\
1.7-5 \\
>5\end{array}$ & $\begin{array}{c}10.8(8.2-17.9) \\
9.8(6.3-11.9) \\
4.7(2.0-6.0)\end{array}$ & & $\begin{array}{l}1.2(0.7-2.3) \\
3.7(1.9-6.9)\end{array}$ & $\begin{array}{l}0.492 \\
<0.001\end{array}$ & & $\begin{array}{l}1.4(0.8-2.7) \\
3.3(1.7-6.3)\end{array}$ & $\begin{array}{l}0.259 \\
<0.001\end{array}$ & \\
\hline CEA & & 0.0075 & & & $0.630(0.562-0.699)$ & & & $0.731(0.666-0.795)$ \\
\hline $\begin{array}{l}<2.5 \\
2.5-13 \\
>13\end{array}$ & $\begin{array}{c}12.9(8.2-19.0) \\
7.7(4.7-10.3) \\
6.3(3.6-7.7)\end{array}$ & & $\begin{array}{l}1.9(1.0-3.5) \\
2.7(1.4-5.2)\end{array}$ & $\begin{array}{l}0.037 \\
0.003\end{array}$ & & $\begin{array}{l}1.6(0.8-3.0) \\
2.3(1.2-4.5)\end{array}$ & $\begin{array}{l}0.156 \\
0.012\end{array}$ & \\
\hline CA 19-9 & & $<0.001$ & & & $0.699(0.638-0.760)$ & & & $0.742(0.679-0.805)$ \\
\hline $\begin{array}{l}<120 \\
120-3960 \\
>3960\end{array}$ & $\begin{array}{c}14.5(8.2-24.3) \\
7.6(5.8-10.8) \\
5.8(2.0-6.8)\end{array}$ & & $\begin{array}{c}2.5(1.3-5.0) \\
5.3(2.5-11.0)\end{array}$ & $\begin{array}{l}0.009 \\
<0.001\end{array}$ & & $\begin{array}{c}2.8(1.4-5.6) \\
5.0(2.4-10.6)\end{array}$ & $\begin{array}{l}0.004 \\
<0.001\end{array}$ & \\
\hline
\end{tabular}

KPS. With HR ranging from 3.0 (unadjusted) to 3.6 (adjusted), pre-treatment CYFRA 21-1 levels had the strongest impact on the end point TTP. This is also reflected by the C index of 0.652 (unadjusted) and 0.713 (adjusted), respectively (see Table $3 \mathrm{a}$ ). Similar data were observed in the OS analysis, where baseline CYFRA 21-1 had the strongest influence in patient survival (Table 3b). The C index for CYFRA 21-1 was most favourable on days $0,7,14,21$ and 28 (unadjusted only). On day 56 (staging), CA 19-9 had the strongest impact on OS with a C index of 0.699 and 0.742 , respectively. The Kaplan-Meier plots for the correlation of baseline CA 19-9, CEA and CYFRA 21-1 levels with TTP and OS are shown in Figure 2 (TTP) and Figure 3 (OS).

Biomarker levels of CYFRA 21-1, CA 19-9 and CEA were also analysed as continuous variables (after logarithmic transformation), as this may potentially represent a more adequate method for the evaluation of a quantitative variable with a broad range (Boeck et al, 2010). Results of these investigations ( $P$-values only) are summarised in Table 4. For univariate analysis on TTP and OS, the absolute marker values ( $\mathrm{Tx})$ as well as the marker kinetics in relation to the pre-treatment baseline level $(\mathrm{Tx}-\mathrm{T} 0)$ are presented. CYFRA 21-1 showed a strong correlation with TTP and $\mathrm{OS}$ at all assessed time points in the univariate evaluation $(P<0.0001$, for details see Table 4). Absolute levels of CA 19-9 had an equal predictive power for TTP as well as OS, whereas CEA showed a lack of statistical significance at only a few time points (day 21 for TTP and day 28 for TTP and OS, see Table 4). CA 19-9 kinetics were significantly correlated with TTP and OS only at time of staging (day 56). However, marker kinetics generally proved to be not as powerful as the absolute marker levels in determining prognosis.

Multivariate analysis. The significant association of CYFRA 21-1 as continuous variable with OS was maintained in the multivariate analysis, confirming the independent prognostic role of CYFRA 21-1 (Table 4). Of note, again only the absolute marker values of CYFRA 21-1 - but not marker kinetics - had a significant impact on OS. Within a second multivariate Cox model including all clinical and biomarker variables (categorised as tertiles) with a $P$-value $<0.1$ in univariate analysis $(n=78)$, only KPS (HR 3.3, 95\% CI 1.7-6.5, $P=0.0003$ ) and pre-treatment CYFRA 21-1 (tertile 2: HR 2.4, 95\% CI 1.1-5.3, $P=0.027$; tertile 3: HR 4.0, 95\% CI $1.7-$ 
A

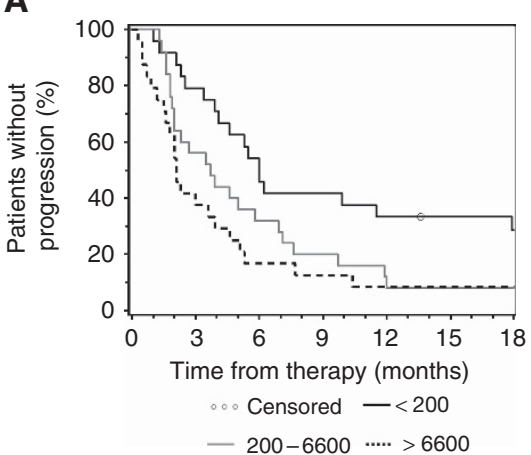

B

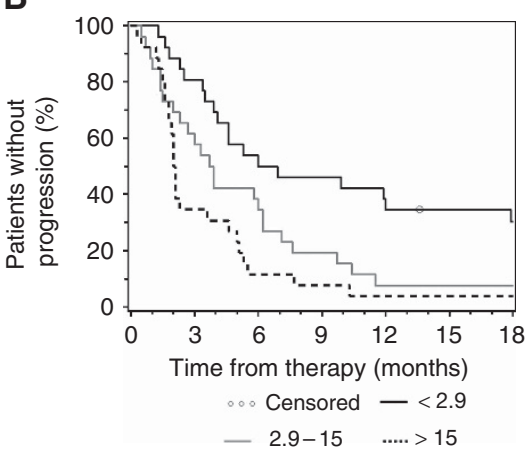

C

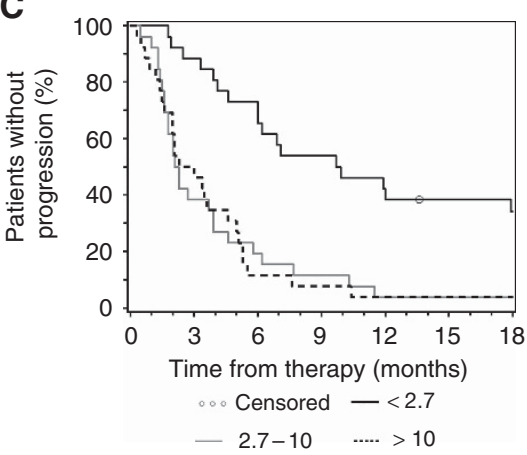

Figure 2. Kaplan-Meier curves for TTP based on pre-therapeutic (A) CA 19-9 $\left(\mathrm{U} \mathrm{ml}^{-1}\right)$, (B) CEA (ng ml $\left.{ }^{-1}\right)$ and (C) CYFRA 21-1 levels (ng ml ${ }^{-1}$ ) (categorised by tertiles).

A

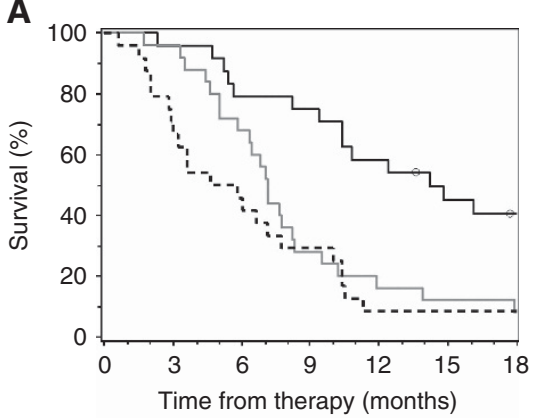

$\therefore$ Censored $-<200$

- 200-6600 ..... > 6600
B

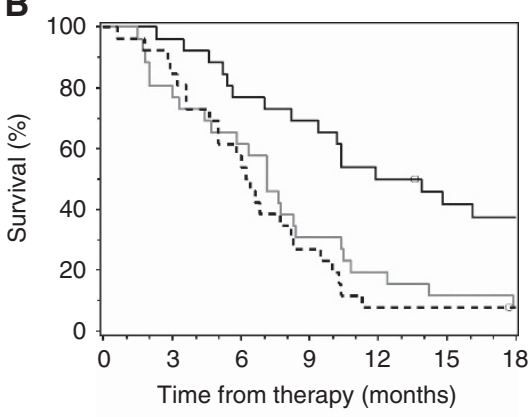

$\ldots$ Censored $-<2.9$

$-2.9-15 \quad \ldots . .>15$

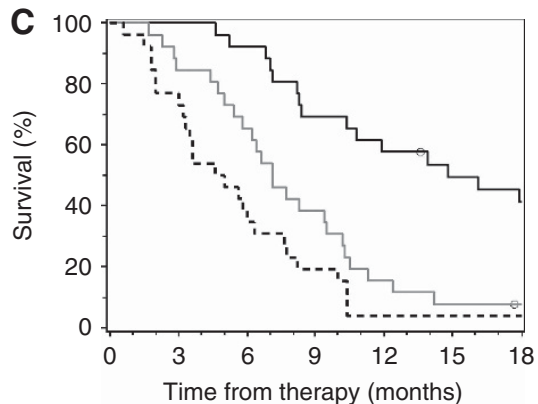

$\therefore$ Censored $-<2.7$

$-2.7-10 \quad \ldots . .>10$

Figure 3. Kaplan-Meier curves for OS based on pre-therapeutic (A) CA 19-9 $\left(\mathrm{U} \mathrm{ml}^{-1}\right)$, (B) CEA (ng ml $\left.{ }^{-1}\right)$ and (C) CYFRA 21-1 levels (ng ml $\left.{ }^{-1}\right)$ (categorised by tertiles).

9.7, $P=0.002)$ - but not CA $19-9$ or CEA - retained their independent prognostic significance for OS. The $\mathrm{C}$ index for that model was 0.769 (95\% CI 0.716-0.822). When this model was reanalysed with only CA $19-9$ syn patients $(n=73)$, the corresponding C index was 0.776 (95\% CI 0.724-0.828), and apart from KPS (HR
3.4, 95\% CI 1.7-6.8, $P=0.0005$ ) and CYFRA 21-1 (tertile 2: HR 3.1, 95\% CI 1.3-7.2, $P=0.0091$; tertile 3: HR 5.1, 95\% CI 1.9-13.7, $P=0.0013$ ), also CA 19-9syn (tertile 2: HR 2.6, 95\% CI 1.2-5.6, $P=0.0144$, tertile 3 : NS) became statistically significant for the end point OS. 
Table 4. Uni- and multivariate analyses for correlation of biomarkers (each analysed as continuous variable; $\left(T_{x}\right)$ indicates the absolute value, $\left(T_{x}-T_{0}\right)$ the marker kinetics in relation to the pre-treatment baseline level) with outcome

\begin{tabular}{|c|c|c|c|c|c|c|c|}
\hline & & \multicolumn{2}{|c|}{ Univariate time to progression } & \multicolumn{2}{|c|}{ Univariate overall survival } & \multicolumn{2}{|c|}{ Multivariate overall survival } \\
\hline Marker & Time (days) & $P(T x)$ & $P\left(T x-T_{0}\right)$ & $P(T x)$ & $P\left(T x-T_{0}\right)$ & $P(T x)$ & $P\left(T x-T_{0}\right)$ \\
\hline \multicolumn{8}{|c|}{ CYFRA 21-1 } \\
\hline & 0 & $<0.001$ & - & $<0.001$ & - & $<0.001$ & - \\
\hline & 7 & $<0.001$ & 0.131 & $<0.001$ & 0.130 & $<0.001$ & 0.542 \\
\hline & 14 & $<0.001$ & 0.262 & $<0.001$ & 0.123 & $<0.001$ & 0.641 \\
\hline & 21 & $<0.001$ & 0.995 & $<0.001$ & 0.261 & $<0.001$ & 0.761 \\
\hline & 28 & $<0.001$ & 0.018 & $<0.001$ & 0.006 & 0.014 & 0.171 \\
\hline & Staging & $<0.001$ & 0.907 & $<0.001$ & 0.413 & $<0.001$ & 0.673 \\
\hline \multicolumn{8}{|l|}{ CEA } \\
\hline & 0 & $<0.001$ & - & $<0.001$ & - & 0.013 & - \\
\hline & 7 & 0.002 & 0.043 & 0.002 & 0.037 & 0.051 & 0.450 \\
\hline & 14 & 0.002 & 0.763 & 0.003 & 0.476 & 0.064 & 0.612 \\
\hline & 21 & 0.062 & 0.260 & 0.043 & 0.207 & 0.273 & 0.954 \\
\hline & 28 & 0.198 & 0.104 & 0.542 & 0.045 & 0.978 & 0.206 \\
\hline & Staging & $<0.001$ & 0.321 & $<0.001$ & 0.236 & 0.036 & 0.006 \\
\hline \multicolumn{8}{|c|}{ CA 19-9 } \\
\hline & 0 & $<0.001$ & - & $<0.001$ & - & 0.005 & - \\
\hline & 7 & 0.001 & 0.919 & $<0.001$ & 0.367 & 0.013 & 0.891 \\
\hline & 14 & 0.002 & 0.229 & $<0.001$ & 0.939 & 0.023 & 0.456 \\
\hline & 21 & 0.002 & 0.154 & $<0.001$ & 0.880 & 0.027 & 0.760 \\
\hline & 28 & 0.012 & 0.173 & 0.008 & 0.473 & 0.145 & 0.590 \\
\hline & Staging & $<0.001$ & $<0.001$ & $<0.001$ & 0.003 & $<0.001$ & 0.001 \\
\hline
\end{tabular}

\section{DISCUSSION}

To date, CA 19-9 is the only established and validated serum biomarker in PC that also is applied routinely in daily clinical practice. A broad variety of other potential biomarkers is currently under investigation, for example, genetic (tissue) biomarkers, epigenetic markers and blood markers including circulating tumour cells (Bhat et al, 2012). Unfortunately, there is still no biomarker available predicting the benefits of a specific treatment (e.g., chemotherapy or targeted therapy) in advanced PC. Early evidence suggests that the tumour KRAS mutational status (for erlotinib treatment) or VEGF pathway genetic variants (for bevacizumab treatment) may serve as such predictive markers. However, these translational data still require prospective validation (Costello et al, 2012; Lambrechts et al, 2012; Boeck et al, 2013).

Thus, the scientific rationale still exists to study other serum tumour markers besides CA 19-9 in order to obtain an easy determinable biomarker that provides prognostic information and also allows patient stratification, for example, within the setting of a clinical trial. Based on data from this prospective single-centre study, CYFRA 21-1 could possibly serve as such a biomarker in advanced PC. We could show that pre-treatment CYFRA 21-1 levels are significantly correlated with TTP and OS, and that CYFRA 21-1 may also predict treatment response to chemotherapy. As patients with high CYFRA 21-1 values before the initiation of palliative chemotherapy are less likely to achieve objective disease control, a more intensive treatment (e.g., with the
FOLFIRINOX regimen) might be considered in such a poorprognosis patient population (Heinemann et al, 2012). Interestingly, the main determinant for response was the absolute CYFRA 21-1 level (at any of the assessed time points) rather than the kinetics during chemotherapy (see Table 2); an observation that also holds true for CA 19-9 and CEA. When CYFRA 21-1 was analysed as continuous variable the strongest prognostic information was again based on the absolute CYFRA 21-1 values at each assessed time point and not on the marker kinetics during treatment (see Table 4).

Most importantly, CYFRA 21-1 was shown to be independent of CA 19-9 in multivariate analysis, and was - apart from KPS - the only significant prognostic factor for OS in a multivariate Cox model. Based on the determined $\mathrm{C}$ indices from our study, it may be postulated that for end point OS CYFRA 21-1 is a potentially more powerful prognostic factor than CA 19-9 (Table 3b). Of note, the $\mathrm{C}$ index for our multivariate Cox model that included all biomarker data as categorised variable was 0.769 , whereas when only CA 19-9syn patients were included in this model, the C index slightly increased up to 0.776 and CA $19-9$ syn remained significant for OS. Thus, it could be concluded that an additional prognostic information from CA $19-9$ is potentially only obtained when patients able the synthesise CA 19-9 are considered. Furthermore, CYFRA 21-1 offers the opportunity to serve as novel serum tumour marker in PC patients who are Lewis antigen negative (about 5-10\%) or who present significant cholestasis - conditions that are well-known limitations for the application of CA 19-9 (Boeck et al, 2006). 
Also, only limited data exist on the role of CEA (a widely used tumour marker in colorectal cancer) in PC (Duffy et al, 2010). Based on the data from this study, CEA may also have a role as tumour marker in pancreatic malignancies because CEA levels at baseline and at staging (day 56) were significantly correlated with objective response and also yielded prognostic information regarding TTP and OS in univariate analysis (Tables 2-4).

The strength of the current biomarker study is based on its prospective design (according to the REMARK guidelines) with the serial (weekly) assessment of marker values throughout the course of the first 2 months of first-line palliative chemotherapy and the use of a unique assay for a centralised marker determination. The investigated patient cohort represents a population comparable to other study populations from PC trials with a median OS of 7.7 months (Table 1). Notably, $45 \%$ of the included patients were treated within a prospective clinical study. The main limitation arises from the fact that this study was conducted at a single high-volume German Cancer Centre and not within a multicentre setting. Thus, an external validation of these provocative novel data on CYFRA 21-1 within a prospective multicentre investigation is recommended, ideally conducted as translational biomarker study accompanying a clinical trial.

In conclusion, CYFRA 21-1 serves as a novel, potent serum biomarker in PC providing independent prognostic information. If other prospective multicentre trials confirm these data, CYFRA 21-1 may have a relevant role in pre-therapeutic prognostic models of clinical factors and laboratory parameters that support patient stratification and may be used for the application of different treatment strategies.

\section{ACKNOWLEDGEMENTS}

This work is part of the doctoral thesis of Christin Wittwer. Reagents for this study were kindly provided by Roche Diagnostics.

\section{CONFLICT OF INTEREST}

PS and SH receive honoraria for conference lectures from Roche Diagnostics. The remaining authors declare no conflict of interest.

\section{REFERENCES}

Berger AC, Garcia Jr M, Hoffman JP, Regine WF, Abrams RA, Safran H, Konski A, Benson 3rd AB, MacDonald J, Willett CG (2008) Postresection CA 19-9 predicts overall survival in patients with pancreatic cancer treated with adjuvant chemoradiation: a prospective validation by RTOG 9704 . J Clin Oncol 26(36): 5918-5922.

Bhat K, Wang F, Ma Q, Li Q, Mallik S, Hsieh TC, Wu E (2012) Advances in biomarker research for pancreatic cancer. Curr Pharm Des 18(17): 2439-2451.

Boeck S, Haas M, Laubender RP, Kullmann F, Klose C, Bruns CJ, Wilkowski R, Stieber P, Holdenrieder S, Buchner H, Mansmann U, Heinemann V (2010) Application of a time-varying covariate model to the analysis of CA 19-9 as serum biomarker in patients with advanced pancreatic cancer. Clin Cancer Res 16(3): 986-994.

Boeck S, Jung A, Laubender RP, Neumann J, Egg R, Goritschan C, Vehling-Kaiser U, Winkelmann C, Fischer von Weikersthal L, Clemens MR, Gauler TC, Märten A, Klein S, Kojouharoff G, Barner M, Geissler M, Greten TF, Mansmann U, Kirchner T, Heinemann V (2013) EGFR pathway biomarkers in erlotinib-treated patients with advanced pancreatic cancer: translational results from the randomised, crossover phase 3 trial AIO-PK0104. Br J Cancer 108(2): 469-476.

Boeck S, Stieber P, Holdenrieder S, Wilkowski R, Heinemann V (2006) Prognostic and therapeutic significance of carbohydrate antigen 19-9 as tumor marker in patients with pancreatic cancer. Oncology 70(4): 255-264.
Costello E, Greenhalf W, Neoptolemos JP (2012) New biomarkers and targets in pancreatic cancer and their application to treatment. Nat Rev Gastroenterol Hepatol 9(8): 435-444.

Duffy MJ, Sturgeon C, Lamerz R, Haglund C, Holubec VL, Klapdor R, Nicolini A, Topolcan O, Heinemann V (2010) Tumor markers in pancreatic cancer: a European Group on Tumor Markers (EGTM) status report. Ann Oncol 21(3): 441-447.

Edelman MJ, Hodgson L, Rosenblatt PY, Christenson RH, Vokes EE, Wang X, Kratzke R (2012) CYFRA 21-1 as a prognostic and predictive marker in advanced non-small-cell lung cancer in a prospective trial: CALGB 150304. J Thorac Oncol 7(4): 649-654.

Fahmueller YN, Nagel D, Hoffmann RT, Tatsch K, Jakobs T, Stieber P, Holdenrieder S (2012) Predictive and prognostic value of circulating nucleosomes and serum biomarkers in patients with metastasized colorectal cancer undergoing selective internal radiation therapy. BMC Cancer 12: 5.

Ferrone CR, Finkelstein DM, Thayer SP, Muzikansky A, Fernandez-del Castillo C, Warshaw AL (2006) Perioperative CA19-9 levels can predict stage and survival in patients with resectable pancreatic adenocarcinoma. J Clin Oncol 24(18): 2897-2902.

Halm U, Rohde N, Klapdor R, Reith HB, Thiede A, Etzrodt G, Mössner J, Keller T (2000) Improved sensitivity of fuzzy logic based tumor marker profiles for diagnosis of pancreatic carcinoma versus benign pancreatic disease. Anticancer Res 20(6D): 4957-4960.

Harrell Jr FE, Lee KL, Califf RM, Pryor DB, Rosati RA (1984) Regression modelling strategies for improved prognostic prediction. Stat Med 3(2): 143-152.

Heinemann V, Haas M, Boeck S (2012) Systemic treatment of advanced pancreatic cancer. Cancer Treat Rev 38(7): 843-853.

Hess V, Glimelius B, Grawe P, Dietrich D, Bodoky G, Ruhstaller T, Bajetta E, Saletti P, Figer A, Scheithauer W, Herrmann R (2008) CA 19-9 tumourmarker response to chemotherapy in patients with advanced pancreatic cancer enrolled in a randomised controlled trial. Lancet Oncol 9(2): $132-138$.

Holdenrieder S, von Pawel J, Dankelmann E, Duell T, Faderl B, Markus A, Siakavara M, Wagner H, Feldmann K, Hoffmann H, Raith H, Nagel D, Stieber P (2009) Nucleosomes and CYFRA 21-1 indicate tumor response after one cycle of chemotherapy in recurrent non-small cell lung cancer. Lung Cancer 63(1): 128-135.

Humphris JL, Chang DK, Johns AL, Scarlett CJ, Pajic M, Jones MD, Colvin EK, Nagrial A, Chin VT, Chantrill LA, Samra JS, Gill AJ, Kench JG, Merrett ND, Das A, Musgrove EA, Sutherland RL, Biankin AV (2012) The prognostic and predictive value of serum CA19.9 in pancreatic cancer. Ann Oncol 23(7): 1713-1722.

Lambrechts D, Claes B, Delmar P, Reumers J, Mazzone M, Yesilyurt BT, Devlieger R, Verslype C, Tejpar S, Wildiers H, de Haas S, Carmeliet P, Scherer SJ, Van Cutsem E (2012) VEGF pathway genetic variants as biomarkers of treatment outcome with bevacizumab: an analysis of data from the AViTA and AVOREN randomised trials. Lancet Oncol 13(7): 724-733.

McShane LM, Altman DG, Sauerbrei W, Taube SE, Gion M, Clark GM. Statistics Subcommittee of the NCI-EORTC Working Group on Cancer Diagnostics (2005) REporting recommendations for tumour MARKer prognostic studies (REMARK). Br J Cancer 93(4): 387-391.

Nakata B, Takashima T, Ogawa Y, Ishikawa T, Hirakawa K (2004) Serum CYFRA 21-1 (cytokeratin-19 fragments) is a useful tumour marker for detecting disease relapse and assessing treatment efficacy in breast cancer. Br J Cancer 91(5): 873-878.

Reni M, Cereda S, Balzano G, Passoni P, Rognone A, Fugazza C, Mazza E, Zerbi A, Di Carlo V, Villa E (2009) Carbohydrate antigen 19-9 change during chemotherapy for advanced pancreatic adenocarcinoma. Cancer 115(12): 2630-2639.

Siegel R, Naishadham D, Jemal A (2012) Cancer statistics, 2012. CA Cancer J Clin 62(1): 10-29.

Vincent A, Herman J, Schulick R, Hruban RH, Goggins M (2011) Pancreatic cancer. Lancet 378(9791): 607-620.

This work is published under the standard license to publish agreement. After 12 months the work will become freely available and the license terms will switch to a Creative Commons AttributionNonCommercial-Share Alike 3.0 Unported License.

Supplementary Information accompanies this paper on British Journal of Cancer website (http://www.nature.com/bjc) 